\title{
Selective Staining and Quantification of Intracellular Lipid Droplets with CBD- Fluor, a New and Versatile Push-Pull Fluorophore
}

S. Israel Suarez, ${ }^{a}$ Caroline C. Warner, ${ }^{b}$ Heather Brown-Harding, ${ }^{a}$ Andrea M. Thooft, ${ }^{b}$

$$
\text { Brett VanVeller, }{ }^{\text {**}} \text { and John C. Lukesh II/a* }
$$

${ }^{a}$ Department of Chemistry, Wake Forest University, Wake Downtown Campus, WinstonSalem, NC 27101, USA. E-mail: lukeshjc@wfu.edu

${ }^{b}$ Depatment of Chemistry, lowa State University, Ames, IA 50011, USA. E-mail:

bvv@iastate.edu

Abstract: A novel lipophilic dye, based on the structures of benzothiadiazole heterocycles, was shown to be an effective fluorescent stain for the imaging of lipid droplets (LDs) within both fixed and live cells. Its high photostability, large Stokes shift, and improved staining and specificity over that of commercial dye Nile Red highlight its great potential as a versatile chemical tool for facilitating LD imaging and research.

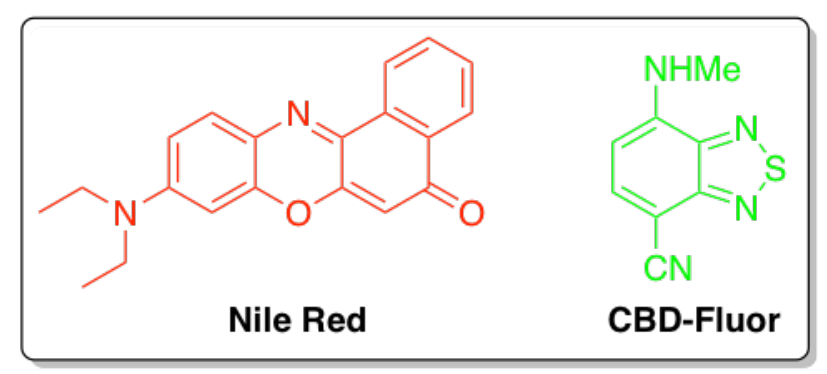

Figure 1. Structures of Nile Red and CBD-Fluor

Lipid droplets (LDs) are highly dynamic cellular organelles involved in the storage and metabolism of neutral lipids, processes which are both tightly regulated and closely 
associated with various metabolic diseases such as obesity and diabetes. ${ }^{1-5}$ To better gauge their relative abundance, size, and distribution, as well as further elucidate their fundamental roles in human health and disease, there remains the need to develop new methods and techniques for the selective detection and visualization of LDs. To this end, lipophilic fluorescent dyes have proven to be invaluable chemical tools for advancing LD research by providing a non-destructive method for the imaging and quantification of LDs in real time-a necessary feature for probing LD dynamics within complex cellular environments. ${ }^{6-13}$

Of the reported LD stains, Nile Red is most commonly used given its commercial availability and long history as an LD marker. ${ }^{14-16}$ However, given its modest photostability and a small Stokes shift-resulting in high levels of noise and background artifacts—-there remains a need to develop new lipophilic fluorophores with enhanced photophysical properties and improved selectivity towards LDs to better assess their primary and secondary biological roles under physiological conditions. ${ }^{17}$ Herein, we report the use of a recently described environmentallysensitive fluorophore, CBD-Fluor (Figure 1), which displays exceptional selectivity for the imaging and quantification of lipid droplets within both fixed and live cells. ${ }^{18}$
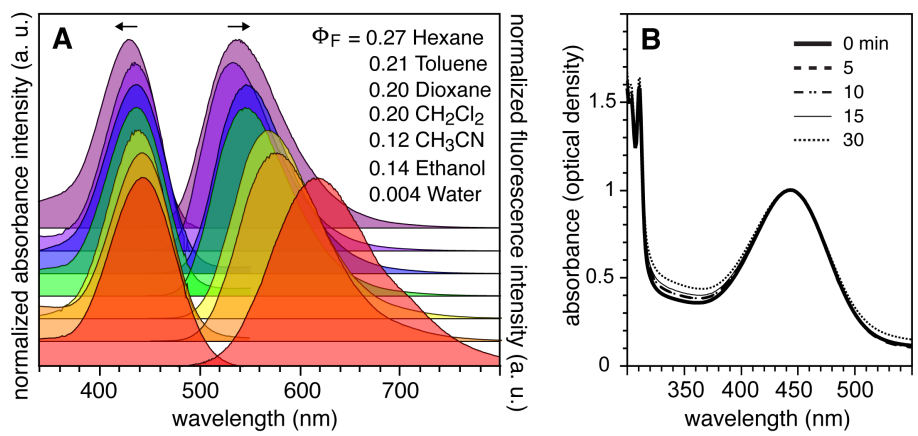

Figure 2: (A) Absorbance and emission spectra for CBD-Fluor showing the solvent polarity and quantum yield effects in various solvents. (B) Photostability of CBD-Fluor demonstrated by an unchanged absorption spectrum under sustained photoirradiation $(\lambda=420 \mathrm{~nm}$, Rayonet) in aerated THF. 
Similar push-pull chromophores based on the benzothiadiazole scaffold have received increasing attention in recent years for bioimaging applications. Their compact structure compared to other polycyclic aromatic dyes is more attractive to prevent disruption of native interactions. Despite this compact structure, however, CBD-Fluor absorbs similarly to larger cell-imaging dyes such as BODIPY and fluorescein (>400 nm, Figure 2A). Moreover, CBD-Fluor displays marked photostability under continuous irradiation (Figure 2B). Dyes related to CBD-Fluor also display large Stokes shifts and environmentally sensitive emission-high emission intensity in non-polar environments and diminished emission in polar environments (Figure 2A). CBD-Fluor, however, was found to display the strongest bias in this turn-on behaviour in non-polar environments. ${ }^{18}$ Thus, we hypothesized that CBD-Fluor would be ideal for applications in lipid staining to address current shortcomings in LD analysis.
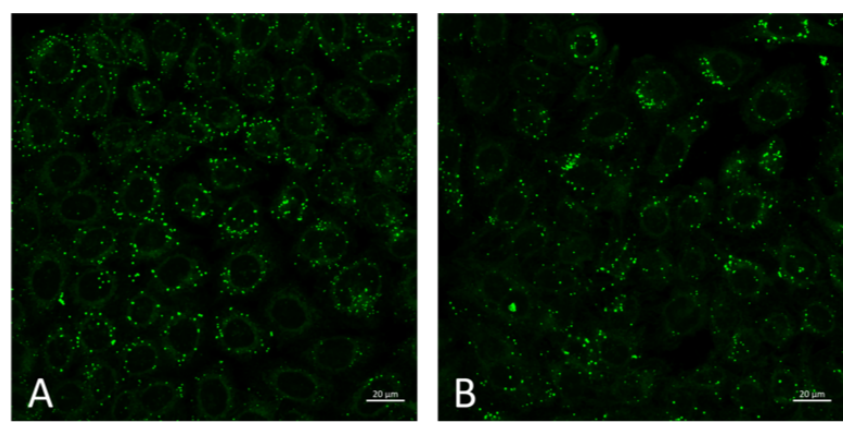

Figure 3. HeLa (human cervical cancer) cells stained for lipid droplets with CBD-Fluor. (A) shows staining with live cells while (B) depicts the staining of fixed cells. Scale bar is set to $20 \mu \mathrm{m}$.

To assess the effectiveness of CBD-Fluor for the selective intracellular staining of LDs, cultured HeLa (human cervical cancer) cells were incubated with CBD-Fluor and imaged using a confocal fluorescence microscope (see supporting information). The obtained images for both fixed and live cells clearly depict 
punctate markings with very little cytoplasmic staining (Figure 3). To ensure that CBD-Fluor was in fact marking spherical structures within the cell and not simply staining lipid structures on the cell's surface, an orthogonal analysis was performed. A total of 14 slices at $13 \mu \mathrm{m}$ was analzyed along the Z-axis (Figure 4). This analysis confirmed that CBD-Fluor was marking spherical structures within the cytoplasm, and that the spherical structures were indeed LDs.

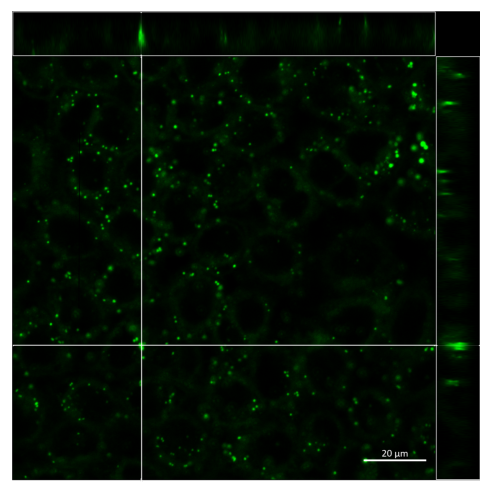

Figure 4. Orthogonal analysis of CBD-Fluor confirms the staining of spherical LDs within the cytoplasm of cells as verified by z-stack analysis. Scale bar is set to $20 \mu \mathrm{m}$.

To further establish CBD-Fluor's LD staining capabilities, we set out to quantify the total number of lipid droplets stained by CBD-Fluor per cell in comparison to that of the well-known lipid marker, Nile Red. Indeed, in live HeLa cells, CBD-Fluor was shown to stain, on average, $25 \%$ more LDs than did Nile Red (Figure 5). Within fixed HeLa cells, CBD-Fluor was even more impressive as it was found to stain $55 \%$ more lipid droplets, on average, than Nile Red. Given their complex structural makeup, one of the few reliable methods for identifying all intracellular LDs is by selectively staining their hydrophobic core with a lipophilic dye. Based on these results, it is likely that CBD-Fluor has either greater lipid affinity or a stronger turn-on response to hydrophobic LDs relative to Nile Red. 
Either way, CBD-Fluor appears to be superior to Nile Red in its sensitivity for detecting lipid droplets within a cellular environment.
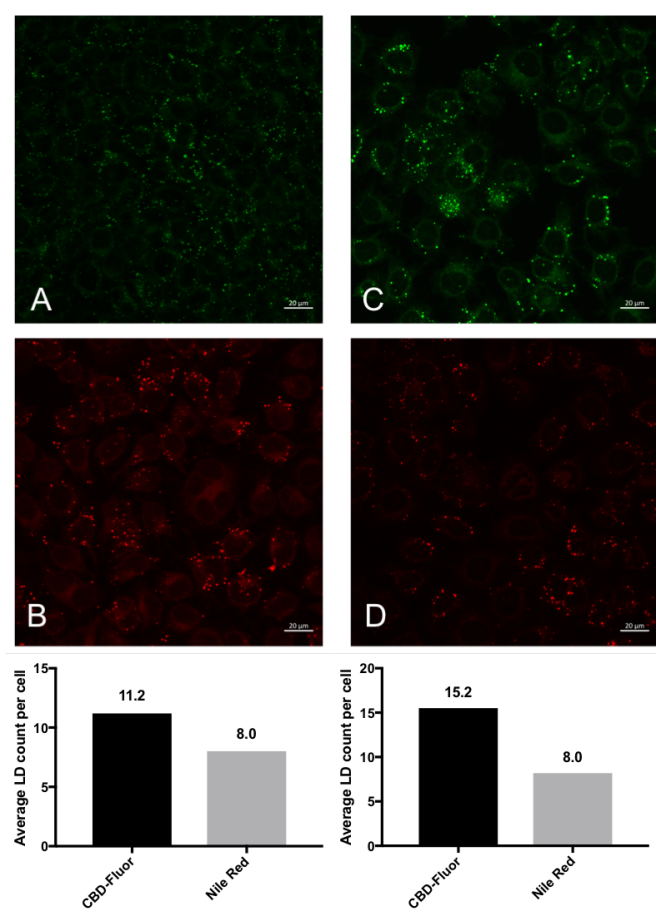

Figure 5. Average lipid droplet count per HeLa over three distinct sampling images. On average, CBDFluor stained more LDs relative to Nile Red. First column: Imaging of live HeLa cells with CBD-Fluor (A) and Nile Red (B). Second column: Imaging of fixed HeLa cells with CBD-Fluor (C) and Nile Red (D). Scale bar is set to $20 \mu \mathrm{m}$.

To further demonstrate the overall utility CBD-Fluor as a versatile LD reporter, we showed that it could also be used alongside other common cellular dyes. Upon co-incubation of CBD-Fluor (green emission) and Hoechst, a common nuclear stain (blue emission), the resulting images in Figure 6 were obtained. An overlay of these signals validated the ease with which CBD-Fluor can be used alongside other cellular markers and its potential for elucidating key interactions between LDs and other cellular compartments. 


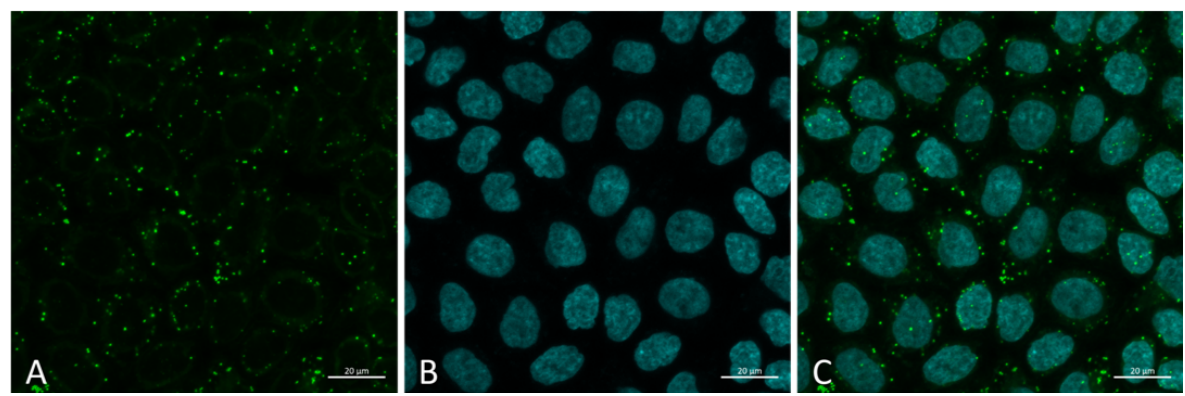

Figure 6. Cellular staining with CBD-Fluor (A, ex: $488 \mathrm{~nm}$, em: $526 \mathrm{~nm}$ ) and Hoechst (B, ex: $405 \mathrm{~nm}$, em: $453 \mathrm{~nm}$ ). (C) Overlay of respective images. Scale bar is set to $20 \mu \mathrm{m}$.

Finally, the relative fluorescence intensities of both CBD-Fluor and Nile Red were also compared in live HeLa cells. The results from this study clearly depict a more favorable signal-to-background ratio for CBD-Fluor (Figure 7A) compared to that of Nile Red (Figure 7B). To better visualize this key result, a 2D histogram was generated for the relative fluorescence intensities of both CBD-Fluor and Nile Red across the same relative area within the cell (Figure 7). The resulting figure visibly shows high fluorescence being given off by CBD-Fluor within the core of LDs with almost zero background fluorescence being emitted outside. In comparison, Nile Red displayed more modest selectivity as a greater than two-fold increase in background fluorescence was calculated. 


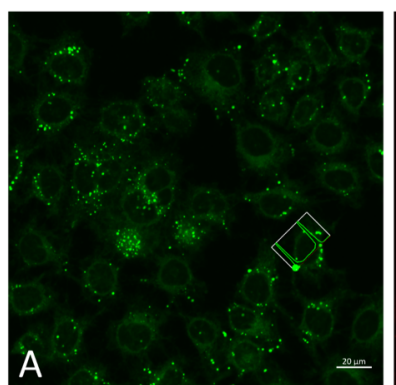

BrettFluor Fl Intensity

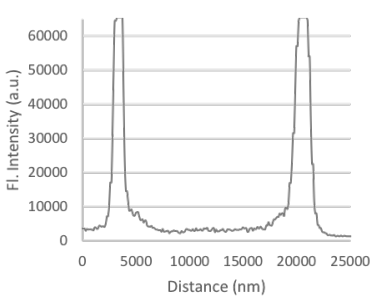

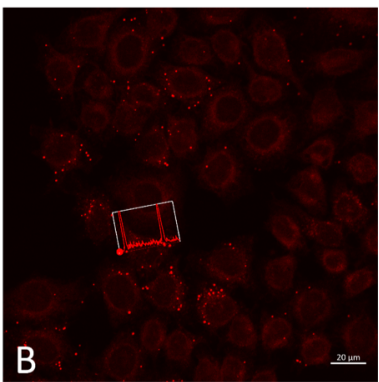

NR Fl Intensity

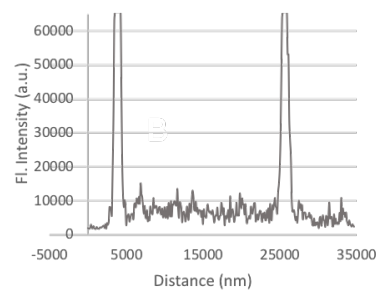

Figure 7. Fluorescence intensities of both CBD-Fluor (A) and Nile Red (B) in live HeLa cells. CBD-Fluor exhibits remarkable selectivity, while Nile Red displays a more modest signal-to-noise ratio. Scale bar is set to $20 \mu \mathrm{m}$.

\section{Conclusion}

In summary, a newly derived push-pull fluorophore (CBD-Fluor) was shown to be highly effective in its ability to stain intracellular lipid droplets in both live and fixed cells. Throughout the course of our investigations we found that CBD-Fluor not only stained, on average, more lipid droplets than did Nile Red, but also displayed more impressive signal-to-background ratios. These attributes, along with its high photostability and large Stoke shift, indicate that CBD-Fluor is a highly effective chemical reporter for LD imaging and research.

\section{Conflicts of Interest}

There are no conflicts to declare

\section{Acknowledgements}

JCL is thankful for financial support from Wake Forest University (start-up funds). BV is grateful for support of this work by the National Science Foundation under 
Grant No. 1848261. This paper is dedicated to Prof. Ronald T. Raines on the occasion of his $60^{\text {th }}$ birthday.

\section{References}

1) Y. Guo, K. R. Cordes, R. V. Farese and T. C. Walther, J. Cell Sci., 2009, 122, 749752.

2) B. Brügger, Annu. Rev. Biochem., 2014, 83, 79-98.

3) P. Shyu, X. F. A. Wong, K. Crasta and G. Thibault, Biosci. Rep., 2018, 38, BSR20180764.

4) M. H. den Brok, T. K. Raaijmakers, E. Collado-Camps and G. J. Adema, Trends Immunol., 2018, 39, 380-392.

5) J. A. Olzmann and P. Carvalho, Nat. Rev. Mol. Cell Biol., 2019, 20, 137-155.

6) L. D. Lavis and R. T. Raines, ACS Chem. Biol., 2008, 3, 142-155.

7) A. B. Neef and C. Schultz, Angew. Chem. Int. Ed., 2009, 48, 1498-1500.

8) J. Spandl, D. J. White, J. Peychl and C. Thiele, Traffic, 2009, 10, 1579-1584.

9) J. Chan, S. C. Dodani and C. J. Chang, Nat. Chem., 2012, 4, 973-984.

10) E. Kim, S. Lee and S. B. Park, Chem. Commun., 2012, 48, 2331-2333.

11) H.-J. Yang, C.-L. Hsu, J.-Y. Yang and W. Y. Yang, PLoS ONE, 2012, 7, e32693.

12) A. Goel, A. Sharma, M. Kathuria, A. Bhattacharjee, A. Verma, P. R. Mishra, A. Nazir and K. Mitra, Org. Lett., 2014, 16, 756-759.

13) A. A. R. Mota, J. R. Correa, L. P. de Andrade, J. A. F. Assumpção, G. A. de Souza Cintra, L. H. Freitas-Junior, W. A. da Silva, H. C. B. de Oliveira and B. A. D. Neto, ACS Omega, 2018, 3, 3874-3881.

14) P. Greenspan, J. Cell Biol., 1985, 100, 965-973.

15) I. A. Boldyrev, X. Zhai, M. M. Momsen, H. L. Brockman, R. E. Brown and J. G. Molotkovsky, J. Lipid Res., 2007, 48, 1518-1532.

16) J. Rumin, H. Bonnefond, B. Saint-Jean, C. Rouxel, A. Sciandra, O. Bernard, J.-P. Cadoret and G. Bougaran, Biotechnol. Biofuels, 2015, 8, 42-57.

17) T. Fam, A. Klymchenko and M. Collot, Materials, 2018, 11, 1768-1786.

18) A. M. Thooft, K. Cassaidy and B. VanVeller, J. Org. Chem., 2017, 82, 8842-8847. 